\title{
Recognizing Visual and Auditory Cues in the Detection of Foreign-Language Anxiety
}

\section{Tammy Gregersen}

This study examines whether nonverbal visual and/or auditory channels are more effective in detecting foreign-language anxiety. Recent research suggests that language teachers are often able to successfully decode the nonverbal behaviors indicative of foreign-language anxiety; however, relatively little is known about whether visual and/or auditory channels are more effective. To this end, a group of 36 preservice English-language teachers were asked to view videotaped oral presentations of seven beginning English-language learners under three conditions: visual only, audio only, and a combination of visual and audio in order to judge their foreign-language anxiety status. The evidence gathered through this study did not conclusively determine the channel though which foreign-language anxiety could be most accurately decoded, but it did suggest indicators in the auditory and visual modes that could lead to more successful determination of behaviors indicative of negative affect.

Cette étude cherche à déterminer par quelle voie l'anxiété face à l'apprentissage d'une langue étrangère est mieux décelée: la voie nonverbale visuelle ou la voie auditive. Les recherches récentes portent à croire que les enseignants de langues réussissent souvent à décoder les comportements nonverbaux qui révèlent de l'anxiété due à la langue étrangère; toutefois, on connaît peu sur l'efficacité de la voie visuelle par rapport à celle de la voie auditive. Pour en apprendre davantage à ce sujet, on a demandé à 36 stagiaires en enseignement de l'anglais de visionner des enregistrements de présentations orales par sept débutants en apprentissage de l'anglais pour juger de leur anxiété face à la langue étrangère. Les stagiaires ont évalué trois formats d'enregistrements: seulement par voie visuelle, seulement par voie auditive, et par voie visuelle et auditive. Les résultats de cette recherche ne permettent pas de conclure de façon irréfutable quelle voie donne une meilleure analyse de l'anxiété liée à l'apprentissage d'une langue étrangère. Toutefois, ils proposent des indicateurs auditifs et visuels qui pourraient aider à mieux déterminer quels comportements révèlent un effet négatif.

\section{Introduction}

Nonverbal behavior, both visual and auditory, provides a plethora of information about language learners' affective states. Visual cues-including kinesics (body movement, gesture, and posture); facial expression and gaze behavior; and nonverbal auditory cues such as pitch, volume, and rate- 
function in concert intentionally or inadvertently to communicate emotion. Many of these internal emotional states can be decoded reliably from nonverbal and vocal behavior, whether the clue is gleaned from a rigid body position or from high-pitched stuttering. Language teachers and researchers in the past have been predominantly concerned with language learners' verbal progress, but because of the crucial role that affect plays in the success or failure of a language learner's linguistic endeavors, classroom and research agendas may benefit from closer scrutiny of nonverbal behavior, particularly in the light of recent research on foreign-language anxiety.

Foreign-language anxiety is a debilitating emotion that is specifically related to the language-learning environment and afflicts language-learners who are apprehensive about communicating in their foreign language and who fear negative evaluation. These learners seem to believe that the person they are while using their first language (L1) cannot be fully communicated in their foreign language (FL). They notice an authenticity gap in their L1 and FL language use and feel at a loss while communicating in a language over which they do not have full command (Horwitz, Horwitz, \& Cope, 1986). Detrimental effects include negative self-talk (MacIntyre \& Gardner, 1991), high absenteeism and procrastination on assignments, overly ambitious performance goals (Gregersen \& Horwitz, 2002), lack of desire to participate in class activities or to volunteer answers in class (Horwitz et al.), and a higher probability of receiving unsatisfactory course grades (Gardner, Smythe, \& Lalonde, 1984). In order to diagnose this condition, language teachers in the past had only these behavioral issues to use as diagnostic tools, which often left many language-anxious students unidentified and feeling abandoned without receiving some of the affective support that might have alleviated their predicament.

Although this list of behavioral manifestations provided language teachers with a point of departure for detecting foreign-language anxiety, preliminary inroads were made with a compilation of nonverbal kinesic behaviors indicative of foreign-language anxiety, which gave teachers actual physical manifestations to look for including more stoical facial expressions and limited eye contact; rigid, closed posture; and use of more self-adaptive gesture than illustrative, speech-related hand movements (Gregersen, 2005). In a later study, evidence suggested that preservice teachers could quite accurately decode the nonverbal behavior indicative of foreign-language anxiety of learners who were at the extreme ends of the anxiety continuum, and that with training, preservice language teachers were able to increase their decoding accuracy (Gregersen, 2007).

Although nonverbal behavioral tendencies indicative of foreign-language anxiety have been described, and the relative ability to decode this behavior with nominal degrees of accuracy has been documented, relatively little is known about whether visual and/or auditory channels are more effective 
signals for detecting language-anxious behavior. That is to say, can negative affect such as foreign-language anxiety be more reliably decoded from auditory cues, visual cues, or a combination of both? To this end, a group of 36 preservice English as a second language (ESL) teachers were asked to view videotaped oral presentations by seven beginning English-language learners-each of whose anxiety status had been previously established through the administration of the Foreign Language Classroom Anxiety Scale, FLCAS (Horwitz et al., 1986), a test that through multiple applications by researchers in the past has been shown to be reliable-under three conditions: visual only, audio only, and a combination of visual and audio-in order to judge the learners' foreign-language anxiety status.

Research suggests that some emotions are more recognizable than others. For example, positive emotion may be more accurately decoded by observers than negative emotion (Zuckerman, Hall, DeFrank, \& Rosenthal, 1976). Variables that may contribute to the correct attribution of emotion are the similarity between the sender's and receiver's language and culture, the context in which the communication occurs, the sex of the interlocutors, and the receiver's affective state (Russell, Bachorowski, \& Fernández-Dols, 2003). Although variability exists, consistency lies in the idea that universally, facial and vocal changes occur everywhere in human interaction and are consistent with the sender's psychological state. Furthermore, most people can infer something about their interlocutor's emotional state from facial and vocal changes (Russell \& Fernández-Dols, 1997).

Although one may find it anecdotally appealing that a combination of visual and auditory channels would result in better decoding of emotion than when limited to the presence of just one or the other, research demonstrates that some messages may be more successfully communicated in one mode than the other. For example, Knapp and Hall (2006) stated that vocal cues may be more effective in the particular instance of communicating anxiety than other communication channels or a combination thereof. However, at the same time, although Knapp and Hall cautioned readers not to rush to generalizations, they stated that generally judgments made using the visual channel, particularly the face, were more accurate than those made with the voice. When receivers of varying cultures, ages, and backgrounds are presented with an emotional expression and asked to indicate which emotion it signals, they agree on the emotion signaled more often than could be achieved by chance, with agreement usually higher for facial expression than vocal expressions (Wallbott \& Scherer, 1986). Our question still remains: Are visual or auditory cues more accurate in decoding the situation-specific emotion of foreign-language anxiety? To answer it most effectively, we turn our attention to each channel individually. 


\section{The Vocal Channel}

Research has demonstrated that vocal behavior communicates emotional states between interlocutors through the use of paralinguistic cues. In summarizing research on vocal cues and emotional expression, Richmond and McCroskey (2004) listed five consistent findings. First, emotions indicating negative feelings are more accurately decoded than positive ones. Second, those listeners who are effective decoders of emotion also tend to be effective encoders of their own emotions when speaking. Third, interlocutors who are successful monitors and controllers of their own emotions are better able to identify the emotions of others through vocal behavior. Fourth, people are better able to recognize emotion through vocal features when their interlocutor is from the same culture, although this does not imply that there is not substantial accuracy even when speakers and listeners are not from the same culture. Finally, detection is more difficult for subtle than for overt emotions.

Research in the area of psychology and communications has rendered some interesting results about emotion and its relation to the auditory channel of communication. Knapp and Hall (2006) used the example of anxiety to exemplify the complexity of using acoustic features to identify emotion. Complexity is exacerbated by contextual cues, the actual words being spoken, accompanying visual nonverbal behavior, individual difference among interlocutors, and the notion that there is more than one way to express any given emotion. For example, some anxiety-ridden speakers may speak more slowly whereas others may speak more rapidly. Whereas some speakers are plagued with nonfluencies and speech disruptions, only certain forms of verbalization are increased and others are not. Cook (1969) demonstrated that although fillers such as er or ah do not increase, sentence changes, repetitions, stuttering, word omissions, sentence incompletions, and slips of the tongue do. Finally, personality dimensions have been found to play a role. For example, Harrigan, Suárez, and Hartman (1994) found that individuals who repress their feelings by denying negative thoughts, impulses, or behaviors are more likely to be judged as more anxious than those who are more aware of their anxiety and take the necessary steps to combat it. Put simply, generally anxious speakers auditorily encode their emotions by raising their pitch, speaking more rapidly, incorporating more speech disturbances (with the exception of $a h$-errors) and silent pauses, and maintaining a breathy voice quality (Argyle, 1990).

\section{The Visual Channel}

Among the visual avenues for communicating emotion-or in the particular case of this study, anxiety-are bodily cues including gesture, posture, and facial expression. Ekman and Friesen (1969) divided gestures into five cate- 
gories: emblems (which have a direct verbal translation like putting the finger to the lips to indicate be quiet), illustrators (which are closely linked to speech and help demonstrate what is being said), regulators (which maintain and regulate interaction), affect displays (which provide information about emotional states), and adaptors (unintentional body movements that are linked with negative attitudes). Although all the categories are important for effective communication in the overall process, the last two-affect displays and adaptors-are the most important in a discussion of the decoding of visual emotional cues.

Affect displays indicate emotional reactions and the strength of those reactions by participating members in a communicative exchange. People may portray an emotion they do not actually feel, or they may repress the expression of an emotion of which they do not want others to be aware. Even though individuals may be conscious of their emotion, affect displays are often unintentional and reveal true emotional states. This unconscious element extends to the gesture category of adaptors, where all such hand movements are defined as highly unintentional. Adaptors are usually responses to boredom or stress and are intimately linked with negative feelings about ourselves or others. These gestures involve self-adaptation where an individual manipulates his or her body by scratching, rubbing, or hair-twisting, among others; object-focused adaptors, which include manipulating something like a pen or twisting a ring; and alter-directed adaptors like crossing one's arms in front of the body, which are designed to create a wall against other interactants (Richmond \& McCroskey, 2004). Gregersen (2005) also found that affect displays and adaptive behavior were common in highly anxious foreign-language students:

When the hands of the anxious participants were not involved in body-focused adaptors ... and object-focused adaptors, the arms were folded in front of the body ... Furthermore, whereas the non-anxious group tended to have their hands folded in their laps when they were not using them in speech-related gestures, members of the anxious group were using their hands in generally "fidgety" ways. (p. 392)

People's posture and how they walk, including limb movements, will also provide information about emotional states, moods, or relationships. Through a relaxed posture, one can communicate openness and willingness to communicate as well as a positive attitude. However, the opposite occurs and communication is restricted when interlocutors send postural cues that limit visibility and increase perceptions of distance. Furthermore, restlessness and reposturing have been associated with feelings of tension, awkwardness, and anxiety (Ekman \& Friesen, 1974). Although increased bodily tension, postural rigidity, closed body positions, and leaning away from interlocutors are signs of reticence and anxiety (Burgoon \& Koper, 
1984), bodily tension is also said to be an effective indicator of the intensity with which an emotion is felt (Richmond \& McCroskey, 2004). The results of this research in the area of communications was also reflected in the results found in the Gregersen (2007) study, where evidence suggested that nonanxious foreign-language learners leaned slightly forward and maintained a relaxed, open body position, whereas anxious learners tended to maintain rigid spines, were tense and closed, and crossed and uncrossed their legs.

Another visual avenue for the detection and/or decoding of anxiety can be found in facial expression, but this can also lead to confusion. One of the problems with using the face as a medium through which to judge a person's emotion is the notion that cultural and social influences have taught people to divorce emotions from facial behavior by using one or more of four facial management techniques: individuals learn to mask or repress the expression of a felt emotion and replace it with something more socially acceptable; when communicators intensify their expressions, they exaggerate what they feel to meet the expressed concern of others; neutralization occurs when an interlocutor essentially eliminates any expression of emotion, as is seen in what is colloquially known as a poker face; finally, a person who deintensifies his or her emotions is one whose facial expression is reduced in intensity because specific circumstances require downplaying what is truly felt.

Although these facial management techniques make a completely accurate recognition of emotion from facial behavior suspect, research has demonstrated that certain emotions are decoded effectively, generally well above chance (Knapp \& Hall, 2006). Gregersen (2005) found that learners with high anxiety were less likely to use facial expressions:

Non-anxious participants generally tensed, grimaced, contorted, and twitched their facial features an average of roughly six times more than the anxious learners. In general, the few movements made by the anxious individuals in this study consisted primarily of nose wrinkling when searching for words. Otherwise, tense facial muscles precluded any other type of facial expression, including smiling. (p. 391)

This preliminary research suggests that using auditory and vocal cues to detect emotion is a complex and often contradictory process. However, because foreign-language anxiety has such debilitating consequences in the language acquisition/learning process, it warrants further attention. To deal with it educators must first recognize the symptoms and identify students who are negatively affected. The purpose of this research is to discover how teacher trainees most accurately decode the nonverbal cues indicative of foreign-language anxiety. Although preservice teachers (as opposed to inservice teachers) have limited exposure to language-learning situations and thus may have greater difficulty in singling out specific language anxiety-related behaviors, they were used in this study to improve the instructional 
strategies of teacher educators at the institution where the research was conducted. To this end, preservice teacher trainees in TESOL were presented with a series of public English presentations by seven beginning languagelearners who were enrolled in an intensive academic English-language program and whose foreign-language anxiety status had been previously established through testing. After being presented with a visual-only clip, an audio-only clip, and a combination clip, each teacher trainee participant was asked to decode the anxiety status of the English-language learner under each videotaped condition. The degree of decoding accuracy was established by comparing each teacher trainee's responses with the learner's actual anxiety status, which had been previously defined by his or her FLCAS score.

\section{Method}

\section{Participants and Procedures}

This study unfolded in two phases. The first was the creation of the videotaped stimulus containing seven English-language learners delivering a public presentation, and the second was the presentation of the stimulus to the preservice teachers who were asked to judge the foreign-language anxiety status of the presenters. For the first phase the researcher selected seven international students from a larger group of 17 who were enrolled in beginning classes of an intensive academic English program at a small United States Midwestern university. This sample consisted of the three most anxious and the four least anxious participants according to their scores on the Foreign Language Classroom Anxiety Scale (Horwitz et al., 1986). Table 1 displays the average FLCAS scores for the 17 learners in the larger population and the scores for the seven individuals participating in the videotaping process.

The seven participants in the videotaped sample were male. Of the 17 English-language learners originally tested with the FLCAS, only two were female and they did not fall within the most or least anxious categories. The

Table 1

FLCAS Scores

\begin{tabular}{lr}
\hline Mean FLCAS Score & 86 \\
\hline High-Anxious Student No. 1 & 109 \\
High-Anxious Student No. 2 & 107 \\
High-Anxious Student No. 3 & 100 \\
Low-Anxious Student No. 4 & 75 \\
Low-Anxious Student No. 5 & 73 \\
Low-Anxious Student No. 6 & 70 \\
Low-Anxious Student No. 7 & 68 \\
\hline
\end{tabular}


preponderance of male English-language learners in this study is consistent with the current population of English language learners in the intensive English program at this university because of the presence of many male students from the Middle East who have come to the US from their countries of origin on scholarships. Although five of the seven participants were from Saudi Arabia, one was also from Russia (low anxious) and another from Brazil (low anxious). In addition, six of the participants were of typical university age and one was an adult student (low anxious).

During the stimulus phase, the English language learners in this study were videotaped by the researcher while giving a public presentation in English in front of their peers and two teachers during a normally scheduled class period. Each student was asked to come to the front of the room and draw a paper from a hat on which was written one of the following questions.

1. Tell us about your best friend. What is his/her name? Where is he/she now? What is he/she doing?

2. Tell us about your weekends. What do you like to do? Where do you go and with whom?

3. Tell us what you would do with a million dollars. What would you buy? Would you give some money to other people? Who and why?

4. Tell us about a city in the US that you want to visit. Why? What do you know about it?

5. Tell us why you are learning English. What will you do with it? Are you going to study more in English? What subject(s)?

6. Tell us about your house in your country. How many rooms are there?

What colors is it painted? Who lives there with you?

In order to not draw attention to who were participants in the study and who were not, all the students in the class, regardless of whether they were included in the study, drew a question and spoke. Each participant was asked to speak for roughly three minutes on his topic, and all complied, although two of the seven learners, both of whom were anxious learners, needed a few prompts from their teacher to keep them talking to meet the three-minute goal.

The 36 participants in the second phase of the study were 12 graduate and 24 undergraduate TESOL (Teaching English to Speakers of Other Languages) majors enrolled at the same Midwestern university. These students were invited to participate in the research project and all agreed. Thirty were female, six were male. Seven were international students (India, Indonesia, Japan, Russia [2], China, Taiwan), and 29 were from the US. Sixteen were between the ages of 19 and 25, and the rest were over 25 years old.

The 36 participants were asked to observe the videotaped presentations by beginning English language learners three times in the second part of the study. Before viewing, participants received three sheets of paper on which 
were written the following instructions: "After viewing and/or listening to the following clips of ESL learners, please respond during the short break between each learner: 1) whether you believe the learner was Anxious (A) or Non-Anxious (N); and 2) the reasons as to why you classified the learner as you did." The first sheet was to catalogue the observations of the participants in response to the seven learners in the clip that had video transmission but no audio; the second sheet was for responses to the clip that had audio transmission but no video; and the third sheet was for responses to the clip that contained both audio and video transmission. The researcher played each clip with 45 seconds between each oral presentation to give observers an opportunity to evaluate and write their responses. Participants then viewed all three clips, classified the seven learners according to their perceived anxiety status, and wrote a short explanation to justify their classification. The researcher then collected and compiled the results.

\section{Results}

Table 2 contains the percentage of observers' accuracy broken down according to the three channels to which they were exposed: video with no audio, audio with no video, and a combination of video and audio. Calculations of the total percentage of accuracy are found in the bottom row.

Table 3 breaks down the percentages of accuracy according to learner and channel. For example, the anxiety status of anxious learner number one was accurately decoded by the observers watching the video with no sound $63.9 \%$ of the time.

\section{Interpretations}

The information in Table 2, which displays the observers' percentage of accuracy according to channel, demonstrates little difference among the observers about their levels of accuracy in determining the anxiety status of the English-language learners giving a public presentation. When viewing the learners with no audio, observers were able to correctly decode the anxiety status of the speaker with $73.9 \%$ accuracy. When the audio portion was played with no video support, observer accuracy decreased roughly four percentage points to $69.6 \%$. Finally, when viewers were exposed to a combination of audio and visual modes, their decoding accuracy was $73.5 \%$. This means that it was negligibly lower (.4\%) than the video with no audio, and slightly higher $(3.9 \%)$ than the audio with no video. To interpret these results, it is necessary to examine the qualitative data found in the tables in the Appendix to discover how observers came to their conclusions. By comparing what accurate decoders were seeing and hearing with what was seen and heard by those who were not as effective in judging the foreign-language anxiety status of the participants, we may come to an improved understand- 
ing of the factors that are the best nonverbal indicators of foreign-language anxiety. By examining factors to which accurate decoders pay attention, we may be able to train teachers better to look for those variables.

Table 2

Observers' Percentage of Accuracy by Channel

\begin{tabular}{|c|c|c|c|}
\hline Observer & Video/No Audio & Audio/No Video & Video and Audio \\
\hline 1 & 72 & 72 & 72 \\
\hline 2 & 72 & 100 & 72 \\
\hline 3 & 44 & 14 & 44 \\
\hline 4 & 100 & 72 & 72 \\
\hline 5 & 100 & 100 & 100 \\
\hline 6 & 58 & 72 & 58 \\
\hline 7 & 72 & 58 & 72 \\
\hline 8 & 100 & 72 & 72 \\
\hline 9 & 100 & 72 & 86 \\
\hline 10 & 72 & 72 & 100 \\
\hline 11 & 72 & 72 & 72 \\
\hline 12 & 72 & 72 & 72 \\
\hline 13 & 72 & 72 & 72 \\
\hline 14 & 44 & 72 & 72 \\
\hline 15 & 72 & 72 & 72 \\
\hline 16 & 72 & 100 & 72 \\
\hline 17 & 72 & 72 & 72 \\
\hline 18 & 72 & 72 & 44 \\
\hline 19 & 100 & 100 & 44 \\
\hline 20 & 72 & 44 & 100 \\
\hline 21 & 72 & 44 & 72 \\
\hline 22 & 58 & 72 & 72 \\
\hline 23 & 72 & 72 & 72 \\
\hline 24 & 72 & 44 & 86 \\
\hline 25 & 72 & 86 & 72 \\
\hline 26 & 100 & 72 & 100 \\
\hline 27 & 72 & 72 & 72 \\
\hline 28 & 72 & 58 & 58 \\
\hline 29 & 100 & 72 & 100 \\
\hline 30 & 44 & 72 & 72 \\
\hline 31 & 44 & 44 & 86 \\
\hline 32 & 72 & 72 & 72 \\
\hline 33 & 44 & 72 & 44 \\
\hline 34 & 100 & 86 & 100 \\
\hline 35 & 86 & 58 & 58 \\
\hline 36 & 72 & 58 & 72 \\
\hline Average Total & 73.9 & 69.6 & 73.5 \\
\hline
\end{tabular}


Earlier research points to the fact that using nonverbal behavior to make judgments about an interlocutor's emotion is a complex and contradictory process. The above-mentioned variables include whether the emotion expressed is positive or negative, the social/cultural and linguistic distance between the interlocutors, the communication context, the sex of the interlocutors, the receiver's affective state, the efficacy of interlocutors in monitoring their emotions, the subtlety of the expressed emotion, idiosyncratic differences, and personality dimensions. However, earlier investigations have also pointed to the notion that even with all these variables, an interlocutor is able to decode correctly the emotional state of his or her conversant with probabilities well above chance, as was also evidenced in this study, with an average accuracy rating among all three modes of $72.3 \%$.

Although relatively little difference was found among the observers exposed to video-only, audio-only, and combined audio and visual channels, in terms of their decoding accuracy for anxious and non-anxious students, Table 3 shows that observers were much more accurate in their decoding of specific learners than of others. For example, although observers were correct $100 \%$ of the time using the combination audio/visual channels for NonAnxious Learner No. 4, they were only $30.6 \%$ correct using the audio/no video mode for Non-Anxious Learner No. 5 . The reasons for this variability can be found in examining the problematic and often contradictory visual and auditory cues that the foreign-language learners were sending and how the receivers interpreted this nonverbal behavior.

\section{Problematic Visual Cues}

One of the most often cited contradictions found in the qualitative data provided by the observers of the video-only segment of the English-language learners was the smiling cue. Whereas the accurate decoders of anxious learners cited behavior like "fake smile" (Anxious Learner No. 1) and "smiled too much" (Anxious Learner No. 2) as an indicator of foreign-language anxious behavior, the inaccurate decoders also used "smiled a lot" to

Table 3

Decoding Accuracy in Mean Percentages of each Learner by Channel

\begin{tabular}{lccc}
\hline Learner & Video/No Audio & Audio/No Video & Video and Audio \\
\hline Learner 1 (A) & 63.9 & 69.5 & 51.7 \\
Learner 2 (A) & 75 & 69.5 & 89.7 \\
Learner 3 (A) & 62.3 & 47.3 & 62.1 \\
Learner 4 (NA) & 94.5 & 94.5 & 100 \\
Learner 5 (NA) & 80.6 & 30.6 & 31 \\
Learner 6 (NA) & 63.9 & 80.6 & 95.1 \\
Learner 7 (NA) & 63.9 & 88.9 & 86.2 \\
\hline
\end{tabular}


justify identifying the same learners as non-anxious. Thus decoding accuracy did not necessarily depend on noting whether the learner smiled or not, but on making an authenticity judgment about whether the smile was genuine. Also, in interpreting this data, one must recognize that cultural differences may play a role in assigning meaning to smiling, both as the sender and the receiver of the smile.

Similarly, judgment of degree was also a factor in evaluating the anxiety status of language learners when the cue concerned eye contact, another nonverbal behavior influenced by culture. Accurate decoders of NonAnxious Learner No. 5 cited "maintained eye contact/did not look at paper" whereas the inaccurate decoders suggested that he had "limited eye contact." Therefore, although both accurate and inaccurate decoders were looking at the same thing, the degree to which observers perceived the cue as within the limits of anxious behavior dictated their response to the nonverbal cue. A similar circumstance arose with the accurate decoders of NonAnxious Learner No. 5, who saw "minimal body rocking" and a "relaxed stance," as compared with the inaccurate decoders, who saw this same behavior as "shifting legs" and "tense posture."

The qualitative data also indicated that observers had difficulty in deciphering which visual elements deserved credence when several conflicting nonverbal cues were present. For example, observers who inaccurately decoded Anxious Learner No. 3 stated in some form that the learner had "relaxed posture" but also "fidgeted some." The observers' mistake was in giving more importance to the relaxed posture and less to the fidgeting. Observers who accurately decoded the participant's hand movements saw these as fidgeting and accordingly rated this learner as anxious.

\section{Problematic Auditory Cues}

After a close examination of the qualitative data following the audio-only segment of the study provided by the inaccurate decoders of foreign-language anxiety, two main elements led to ineffective judgments. Many of the inaccurate observers confused low volume with anxiety. This was particularly the case with Non-Anxious Learner No. 5. The accurate decoders mentioned his "natural pace" and his "quiet but steady" flow. Inaccurate observers equated low volume with uncertainty and remarked on his hesitancy and speaking softly.

The second major audio-only stumbling block concerned an inability to separate issues of fluency from foreign-language anxiety. This confusion permeated almost all the commentaries made by inaccurate decoders, who pointed to the learners' overuse of fillers and slower pace (both Non-Anxious Learners 5 and 6). In general, the inaccurate decoders remarked on fluency when trying to justify their analysis of anxious learners. It must be noted that anecdotally one would hope that as these preservice teachers gained more 
classroom experience, their ability to distinguish fluency from accuracy would improve.

Finally, the ability to distinguish anxious from non-anxious learners using audio cues was hampered by the same notion of degree found in the visual cues. For example, the observers who were able to assess the nonanxious status of Learner No. 7 accurately heard his "good inflection/intonation," whereas the inaccurate assessors heard only a "monotone" quality in his voice. Thus the question: When does a lack of inflection turn into monotone? Accurate assessment again demands an analysis of degree, which would most probably improve through experience as teachers gain the knowledge that appropriate intonation is a natural part of language learning.

\section{What They Got Right}

Although there is much to learn from examining where the observers went wrong, much is also to be gained from what they got right. Observers who accurately decoded the visual behavior indicative of foreign-language anxious students noted that they fidgeted either with their hands or with a paper held in their hands; that they rocked back and forth, shifting from foot to foot; that they smiled, but not genuinely; that they made limited eye contact; and that they had inexpressive faces. Accurate observers of nonanxious students commented that they used their hands for natural and casual gesturing, that they had an open and relaxed body position, that they maintained eye contact, and that they laughed and smiled naturally.

From an auditory perspective, the behaviors cited by effective observers, which aided in accurate decoding of anxious learners, included excessive pausing, too many fillers, monotonous voice, inappropriate laughter, a need for teacher's prompting, and too much repetition. Auditory cues that were effectively used to accurately decode non-anxious learners included a strong, loud voice; expressiveness; variation in speed, pitch, and stress; and a natural pace and fluency. However, as a cautionary note one must consider that these vocal behaviors are not exclusively related to anxiety and that issues like intonation, pausing, and pace are challenges for most language-learners and not necessarily indicative of anxiety.

\section{Conclusion}

Although the evidence gathered through this study did not conclusively determine the channel through which foreign-language anxiety could be most accurately decoded, it did suggest indicators in the auditory and visual modes that could lead to more successful determination of behaviors indicative of negative affect. Although the data demonstrated minimal differences in preservice English language teachers' ability to decode visual-only, auditory-only, and a combination of visual/auditory cues to assess the 
foreign-language anxiety status of English language learners, the evidence suggests that in each channel are specific behaviors that when targeted by observers lead to greater nonverbal decoding accuracy. For a more complete catalog of nonverbal cues that are indicative of the presence of foreign-language anxiety, see Gregersen (2005).

Foreign-language anxiety can often be mistaken for other classroom challenges such as lack of interest or motivation on the part of students. Because foreign-language anxious students often avoid participation in the classroom and sometimes skip classes, teachers may misinterpret these students' behaviors and thus not address the real problem correctly. Among the benefits that this study may bring about are a sensitivity to and awareness of the behaviors that accompany foreign-language anxiety. With more accurate interpretations of nonverbal behavior in the language classroom, instructors will be better able to recognize students who suffer from this inhibition and address the specific needs of these individuals. A teacher's cognizance of an individual learner's anxiety will be the starting point for dealing with it.

\section{The Author}

Tammy Gregersen, an English-Spanish bilingual, earned her doctorate in linguistics at the Universidad Católica de Valparaíso in Chile. She is currently a professor of TESOL at the University of Northern Iowa where she specializes in second-language acquisition methodology, individual differences, and K-12 instruction. She has published in such journals as the Modern Language Journal, Foreign Language Annals, Teacher Trainer, Language Teaching Research, Reflections on English Language Teaching, International Journal of Teaching and Learning in Higher Education, Lenguas Modernas, and Signos She has also traveled extensively, presenting papers, conducting workshops, and teaching in international postgraduate programs in Spain, France, Portugal, Russia, Poland, and Chile.

\section{References}

Argyle, M. (1990). Nonverbal vocalizations. In J. DeVito \& M. Hecht (Eds.), The nonverbal communication reader (pp. 253-265). Prospect Heights, IL: Waveland Press,

Burgoon, J., \& Koper, R. (1984). Nonverbal and relational communication associated with reticence. Human Communication Research, 10, 601-626.

Cook, M. (1969). Anxiety, speech disturbances, and speech rate. British Journal of Social and Clinical Psychology, 8(1), 13-21.

Ekman, P., \& Friesen, W. (1969), The repertoire of nonverbal behavior: Categories, origins, usage and coding, Semiotica, 1, 49-98.

Ekman, P., \& Friesen, W. (1974). Detecting deception from the body or face. Journal of Personality and Social Psychology, 29, 288-298.

Gardner, R., Smythe, P., \& Lalonde, R. (1984). The nature and replicability of factors in second language learning (Research Bulletin No. 605). London, ON: University of Western Ontario. (ERIC Document Reproduction Service, No. ED 248693)

Gregersen, T. (2005). Nonverbal cues: Clues to the detection of foreign language anxiety. Foreign Language Annals, 38, 388-400.

Gregersen, T. (2007). Breaking the code of silence: A study of teachers' nonverbal decoding accuracy of foreign language anxiety. Language Teaching Research, 11, 209-222. 
Gregersen, T., \& Horwitz, E. (2002). Language learning and perfectionism: Anxious and non-anxious language learners' reactions to their own oral performance. Modern Language Journal, 86, 562-570.

Harrigan, J., Suarez, I., \& Hartman, J. (1994). Effect of speech errors on observers' judgments of anxious and defensive individuals. Journal of Research in Personality, 28, 505-529.

Horwitz, E., Horwitz, M., \& Cope, J. (1986). Foreign language classroom anxiety. Modern Language Journal, 70, 125-132.

Knapp, M.L., \& Hall, J. (2006). Nonverbal communication in human interaction (6th ed.), Belmont, CA: Thomson Wadsworth.

MacIntyre, P., \& Gardner, R.C. (1991). Investigating language class anxiety using the focused essay technique. Modern Language Journal, 75, 296-304.

Richmond, V., \& McCroskey, J. (2004). Nonverbal behavior in interpersonal relations. Boston, MA: Pearson.

Russell, J., Bachorowski, J., \& Fernández-Dols, J. (2003). Facial and vocal expressions of emotion. Annual Review of Psychology, 54, 329-349.

Russell, J., \& Fernández-Dols, J. (1997). The psychology of facial expression, New York: Cambridge University Press.

Wallbott, H., \& Scherer, K.R. (1986). Cues and channels in emotional recognition. Journal of Personality and Social Psychology, 5, 690-699.

Zuckerman, M., Hall, J., DeFrank, R., \& Rosenthal, R. (1976). Encoding and decoding of spontaneous and posed facial expressions. Journal of Personality and Social Psychology, 34 , 966-977.

\section{Appendix}

The following three tables were compiled from the qualitative data gathered from the observers as they watched and/or listened to the video clips of the English language learners. Only those comments that were expressed by at least three observers are included below.

Table 4

Qualitative Data from Observers of the Video Clip With no Audio

During the video/no audio experience,

Participants who decoded

correctly consistently

observed:

Anxious Learner No. 1 (1)

Anxious Learner No. 2 (2)
*Kept ruffling his paper

${ }^{*}$ Rocking body

movements/Shifts from foot

to foot

${ }^{*}$ Fake smile

${ }^{*}$ Rarely looked up from

paper/Poor eye contact

*Moved hands in and out of pockets/Plays with clothing

${ }^{*}$ Rocked from side

to side/Lots of leg movements

*Smiled too much

${ }^{*}$ Did not look at the

audience-looks to teacher
Participants who decoded incorrectly consistently

observed:

*Smiled a lot

*Referenced paper when

needed

*Did not gesture

*Stood up straight

*Made some eye contact 
for approval, and down at the paper in his hand

*Looked down at paper

*Small, but limited hand movements/Fidgeted with paper

${ }^{*}$ Rocked back and forth, but otherwise rigid/stiff/awkward

*Took big breaths while talking *Limited expression
Non-Anxious Learner No. 4 (3)

Non-Anxious Learner No. 5 (4)

Non-Anxious Learner No. 7 (7)

*Did not play with his hands *Open, relaxed body posture * Good variety of purposeful large natural hand gestures to illustrate speech *Maintained eye contact and did not look at the paper *Vivid facial expression *Walked around the front of the room *Laughed and smiled naturally *Maintained eye contact/Did not look at paper *Minimal body rocking/Relaxed stance

*Natural gestures

*Smiled a lot

Non-Anxious Learner No. 6 (6)

*Head-nodding

*Natural, casual gestures

*Maintained eye contact/Did not look at paper

*Smiled naturally ${ }^{*}$ Relaxed body position

*Moved his head

*Made eye contact

*Used natural gestures

*Expressive face

${ }^{*}$ Relaxed posture
*Smiled a lot/laughed

*Facially animated

${ }^{*}$ Relaxed body posture with hands in pockets

*Looked confused, but not anxious

*Head nods

*Eyebrows raised

*Relaxed posture

*Good eye contact

*Fidgeted some, but seemed calm

*Looked less anxious as he continued

*Relied too heavily on gesture
*Limited eye contact

*Shifting legs

*Tense posture

*Fidgeting

*Smiled throughout

*Stiff

${ }^{*}$ Avoided eye contact

${ }^{*}$ Fake smiling

${ }^{*}$ Right arm did not move

*Body swayed/Standing

awkwardly

${ }^{*}$ Rapid hand movements

*Scratched different body parts (nervous tic)

*Hand fidgeting

*Swayed back and forth

*Swallowed a lot 


\section{Table 5}

Qualitative Data From Observers of the Audio Clip With No Video

During the audio/no video Experience,

Participants who decoded correctly consistently observed

Anxious Learner No. 1 (1)

Anxious Learner No. 2 (2)

Anxious Learner No. 3 (5)

Non-Anxious Learner No. 4 (3)

Non-Anxious Learner No. 5 (4)

teacher/prompting

*Stuttered
*Paused too often, stilted flow

${ }^{*}$ Fast speech after pauses

*Used too many fillers

*Monotonous voice

*Laughed inappropriately

*Pitch fluctuations

*Too many pauses/Searching for words

*Too many fillers

*Laughed nervously

${ }^{*}$ Repeated himself

*Needed help from

*Shaky voice

*Monotone

*Many fillers

*Slow speech; too many

pauses;

sounded hesitant, uncertain

*A lot of repetition

*Unsure of grammar

*Strong, loud voice

*Very expressive voice;

intonation; vivacious;

animated; enthusiastic;

assertive

*Variety in speed and stress

*Steady pace

*Did not need teacher

prompting

${ }^{*}$ Rarely paused

*Not embarrassed to make

mistakes; circumlocuted

${ }^{*}$ Pitch variety

*Natural pace

*Spoke fluently with few pauses or stuttering

${ }^{*}$ Quiet but steady
Participants who decoded incorrectly consistently observed

*Stable, not shaky

*Even pitch

*Relaxed pacing, as if telling a story

*Spoke loudly

\author{
*Laughed, but not nervously \\ ${ }^{*}$ Pitch variety; inflection \\ *Paused, but to choose words \\ carefully, more a lack of \\ proficiency; \\ ${ }^{*}$ Normal rate of speech \\ *Enjoyed the topic \\ *Voice was not \\ constant-almost "desperate"
}

*Spoke softly, weakly, uncertainly; hesitant *Used pauses and fillers ${ }^{*}$ Forgot a lot of words; Did not know what to say Observers equated low volume with uncertainty; everyone mentioned volume. 


\begin{tabular}{|c|c|c|}
\hline Non-Anxious Learner No. 6 (6) & $\begin{array}{l}\text { *Variations in speed and } \\
\text { intonation } \\
\text { *Higher language proficiency } \\
\text { *Few pauses or hesitations } \\
\text { *Assertive volume } \\
{ }^{*} \text { Calm, steady voice } \\
\text { *Greeted the audience }\end{array}$ & $\begin{array}{l}\text { *Voice was shaky } \\
{ }^{*} \text { Needed teacher prompt } \\
\text { *Used fillers }\end{array}$ \\
\hline Non-Anxious Learner No. 7 (7) & $\begin{array}{l}\text { *Natural pauses } \\
{ }^{*} \text { Good inflection/intonation } \\
{ }^{\star} \text { Steady fluency } \\
\text { *Laughed } \\
{ }^{*} \text { Spoke calmly, assertively } \\
\text { and confidently } \\
{ }^{*} \text { Provided details }\end{array}$ & ${ }^{*}$ Monotone \\
\hline
\end{tabular}

\section{Table 6 \\ Qualitative Data from Observers of the Clip Containing Both Video and Audio}

During the audio and video experience,

Participants who decoded correctly consistently observed:

Anxious Learner No. 1 (1)

Anxious Learner No. 2 (2)

*Stiff posture

${ }^{*}$ Restricted movements

*Long pauses

*Looked at notes

*Sounded hesitant

*Spoke quietly
*Voice sounded tight/tense

*Hands in pockets/played *Spoke loudly

with clothing

${ }^{*}$ Repeated himself

*Long pauses (may be due to language problems)

*Trouble finding words

*Uneasy movements

${ }^{*}$ Rocked back and forth

*Nervous smiling/laughing

*Needed prompting

*Wanted to change the

question

*Made jokes

*Hands in pockets but also

gestured appropriately

*Smiled

*Paused often

*Used a lot of fillers

*Spoke quietly

*Unnatural stance
Participants who decoded incorrectly consistently observed:

*Spoke briefly/clipped

*Stable tone

*Smooth speech

${ }^{*}$ Quiet voice

${ }^{*}$ Controlled fidgeting 
*Fidgeted with paper

*Shifted his weight

*Repeated himself

$\begin{array}{ll}\text { Non-Anxious Learner No. } 4 \text { (3) } & \text { *Used large gestures to } \\ & \text { illustrate points } \\ & \text { * } \text { Made eye contact } \\ & \text { *Natural speaking style } \\ & \text { *Vivid facial expression } \\ & \text { *Very animated } \\ & \text { *Good pace } \\ & \text { *Pitch variation } \\ & \text { *Used humor } \\ & \text { *Used L1 to compensate for } \\ & \text { lack of word retrieval } \\ & \text { *Was putting on a show } \\ & \text { *Smiled and laughed } \\ \text { Non-Anxious Learner No. 5 (4) } & \text { *Good eye contact } \\ & \text { *Steady voice }\end{array}$

Non-Anxious Learner No. 6 (6) *Good eye contact *Smiled genuinely/makes jokes

*Self-corrected errors

*Good pace

${ }^{*}$ Relaxed posture

*Expressive voice

${ }^{*}$ Responded well when audience did not understand

Non-Anxious Learner No. 7 (7) $\quad$ *Lack of vocabulary did not bother him

${ }^{*}$ Relaxed facial expression

*Used a lot of gestures

${ }^{*}$ Genuine smile

*High language proficiency

*Good eye contact

*Spoke assertively and confidently

*Provided many details
${ }^{*}$ Relaxed posture

*Good eye contact

*Calm, slow

*Made jokes

*Open foot position

*Spoke quietly

*Limited eye contact

*Stuttered

*Was hard to understand

*Looked at floor-limited eye

contact ${ }^{\star}$ Shifted from foot to foot

${ }^{*}$ Restricted gestures

*Lacked intonation

*Fidgeted with paper

*Used fillers

*Seemed confused

*Swayed

*Stuttered

*Hesitated

*Looked at the ceiling

*Fidgeted with hands 